miliary tuberculosis and filled with fluid; the /go through their whole course completely flaccid caseous type, in which there are large masses and with diminished or absent reflexes, and, in of newly formed tuberculous tissue in the mesen- babies, even without bulging of the fontanelle. tery and omentum, with sometimes a small It is true that a very few patients have apparamount of fluid; and the adhesive type, in which ently recovered from tuberculous meningitis. the intestines are all bound together, and in The number of cases is so small, however, that we which there is no fluid. The physical signs are not justified, it seems to me, in holding out must evidently be very different in these three any hope whatever of recovery in tuberculous types. The prognosis is far better in tubercu- meningitis. We must remember, however, that lar peritonitis than was formerly supposed. other conditions, such as syphilitic meningitis Many cases recover. It is impossible, however, and encephalitis, may strongly resemble tuberto tell in the beginning whether an individual culous meningitis in their symptomatology and patient will do well or not. Personally, I am that the findings in the cerebrospinal fluid in opposed to operation, except in the ascitic form, these conditions are the same, except for the when it has failed to yield to medical treatment and repeated tapping, and in the other forms, when there is obstruction. In my opinion the prognosis is as good, or better, under medical treatment than with operation. Incidentally, in doubtful cases, the demonstration of disease in the testicle is very strong evidence in favor of tuberculosis of the peritoneum.

'There seems to be a good deal of misunderstanding as to the use of fat in the food in these cases. It used to be given routinely in order to improve the nutrition, but then we were told that, on account of the involvement of the mesenteric lymph nodes, fat could not be absorbed and consequently did harm. 'The truth of the matter is that in some cases the mesenteric glands are involved and fat cannot be absorbed, while in others they are not involved and fat can be absorbed. The only way to determine whether they are involved or not, is by an examination of the stools. If the stools show that fat is not absorbed, the fat should be cut out of the diet. If they show that fat is absorberl, fat should be given and pushed.

Tuberculosis of the mesenteric and retroperitoneal glands is not uncommon. It may give rise to acute symptoms, suggesting those of appendicitis or acute intestinal obstruction. This possibility should never be forgotten in the differential diagnosis of acute abdominal conditions in childhood.

Meningeal Tuberculosis. I know of no condition in which it is harder to make an early diagnosis than tuberculous meningitis. There is nothing characteristic about the early symptoms. Vomiting without evident cause, headache, and disinclination to play are perhaps among the earliest symptoms, but these symptoms are common to many other conditions. The only way in which a physician can always make an early diagnosis is to speak of it as a possibility whenever a child is sick and there is no very evident cause for the illness. I fear, however, that if a physician did this he would soon lose all his patients. It must not be forgotten that the flaccid type of tuberculous meningitis is almost, if not quite, as common as the type with spasm, rigidity of the neck and convulsions, especially in infancy. I have seen many cases of tuberculous meningitis absence of tubercle bacilli. There is always a chance for error, therefore, unless tubercle bacilli have been found in the spinal fluid.

I am confident that lumbar puncture has no curative action in these cases. In the spastic cases and in the cases with marked symptoms of cerebral irritation, lumbar puncture relieves them more, however, than any other method of treatment.

\title{
SURGICAL TUBERCULOSIS.
}

The physician must always bear in mind the possibility of surgical tuberculosis. He must remember that backache and pains in the legs may be due to tuberculosis of the spine. He must not forget that pains in the extremities are not always due to rheumatism, but may be due to tuberculosis of the joints. In fact, he should make the examination of the spine and extremities a part of his routine physical examination, in order that he may never miss conditions of this sort.

\section{TREATMENT}

In my opinion the preventive treatment of tuberculosis is by far the most important. When tuberculosis has developed, the treatment consists of rest, food, fresh air, both day and night, and all the sunlight that there is. I have had but little experience with the Rollier treatment, but from what I have seen of it, I am eonvineed that it is very useful in tuberculosis of the bones and of the peritoneum. I have no confidence whatever in any drug treatment of tuberculosis, except for the relief of symptoms. I have had no personal experience with the tuberculin treatment. What I have seen of it, however, has been most disappointing, for it has seemed to me that it did more harm than good. My impression is that it is being used much less at present than it was a few years ago.

I'Uberculosis in Children from the StandPOINT OF THE ORTHOPEDIST.

By Joer E. Goldthwatt, M.D., Boston.

IT is hard to know what to say at a meeting of this sort, speaking as an orthopedist, on a subject as general as this. You certainly do not wish detailed accounts of the local treatment of 
bone and joint disease, and a few general statements of principle seem best to meet the need. The orthopedist has quite generally come to recognize that tuberculosis of bones and joints is due very largely to low general resistance of the individual. 'This being the case, we have two distinct features to observe in our treatment. One is to treat the part locally where the disease manifests itself, and the other is to see that everything is done that is possible to improve the general condition of the individual. The time was when the wards of our children's hospitals were filled with patients having hip or spine disease where the entire emphasis was put upon the treatment of the local condition. We have gone far beyond that point. The general treatment should receive at least equal attention.

It seems to me that we can pass over the local treatment by saying that in tuberculosis of the bones or joints, the things that the orthopedist aims to do are first to prevent deformity, or correct deformity if it has already occurred, and second, to put the part in the position of physiological rest-then allowing function in so far as the part will tolerate it. Anything that the individual can do with a diseased part (the individual, not someone else) we need have very little fear of doing harm. If the child can walk about once, the part is properly protected-let him walk about. If it is very acute, the child will instinctively not walk about. As fast as the child is able to use that part, it is to be encouraged. Use stimulates circulation, encourages bone repair and does the very thing we ought never to lose sight of-which is to stimulate the cells about the diseased portion, so that they will be stronger and able to control the disease rather than to weaken those parts by too. great inactivity.

For the general condition the things which we are now doing have for their purpose the increasing, in so far as is possible, the general resistance and therefore the more perfect control of the local manifestation of the disease. This applies not only to tuberculosis, it applies to all sorts of infections, - the principles are practically the same. The thing that has helped us most in this connection and has given us something that we can speak about definitely because it is easily checked, has to do with the way the diaphragm is used. It seems to me that this is one of the most important single features of the physiology of the human being. If the diaphragm is down at the level of the twelfth rib and does not move-the circulation of the abdominal organs must suffer. Normal respiration involves the diaphragm and the normal circulation in the abdomen demands the same thing. Ideal respiration means the diaphragm high with slight movement of the chest-slow respiration and slow pulse. The diaphragm not only helps in the respiratory development, but it also pumps the blood from the abdomen and the legs back to the heart. 'There is no other mechanism to accomplish this. With the diaphragm low, the cireulation as well as the respiration will be interfered with and the general vitality will suffer.

Until you get the diaphragm so that it acts freely, you have not done all that you should do to improve the general resistance of the individual.

In trying to build up your individual to the best health-in trying to bring up their reserve so they can avoid tuberculosis-you have tangi. ble things which you can check. Certainly there is more than just the microscope or the stethoscope in understanding these conditions.

Training for this physiological test is the sort of thing our preventoria should be doing. Get the children to stand properly. They will look better, but they will be more normal physiologically, and have greater resistance to tuberculosis or any other disease.

Tuberclilosis in ('HIILDREN From tIIE Stand POIN'T OF 'THIE INTERNIST.

By Henry D. Chadwick, M.D., Westfield, Mass.

IN considering the subject of tuberculosis in children it is necessary to divide them into groups as this disease varies very much in its manifestation according to the age of the child. There are three distinct types of tuberculosis in children:- the general or infantile, the hilum or juvenile, and the apical or adult form.

The infantile form in its primary stage assumes the characteristics of an acute, infectious fever, and progresses rapidly to a fatal termination, as general miliary tubereulosis, meningitis or bronchopneumonia. This is the usual course when infection occurs before the age of two years. From this age on the cause of infection is gradually modified by the production of a steadily increasing immunity. The tissues gradually acquire the power to react and the bacilli become fixed in the lymph nodes or more rarely in the epiphyses of the long bones or in the bodies of the vertebrae. After children have eached the age of five they rarely develop the in. fantile type of tuberculosis.

From five to twelve the lymphoid type of tuberculosis largely predominates. This period is a golden interval of low mortality between the infantile form on the one hand and the adult pulmonary form on the other, both of which have very high death rate. This tendency to focalize in the lymph nodes is the outstanding characteristic of juvenile tuberculosis. This was very apparent a few years ago when cervical tuberculous adenitis was such a common affliction in the children that came to the surgical outpatient departments. It is not so today largely because of the activities of the throat specialists in removing adenoids and diseased tonsils. Also the dentists must be given some of the credit because of the progress of dental hygiene. 